\title{
Analog Characterization of Low-Voltage MQW Traveling-Wave Electroabsorption Modulators
}

\author{
Bin Liu, Member, IEEE, Jongin Shim, Member, IEEE, Yi-Jen Chiu, Adrian Keating, Member, IEEE, \\ Joachim Piprek, Senior Member, IEEE, and John E. Bowers
}

\begin{abstract}
In this paper, high-speed traveling-wave electroabsorption modulators (TW-EAMs) with strain-compensated InGaAsP multiple quantum wells as the absorption region for analog optical links have been developed. A record-high slope efficiency of $4 / \mathrm{V}$, which is equivalent to a Mach-Zehnder modulator with a $V_{\pi}$ of $0.37 \mathrm{~V}$ and a high extinction ratio of $>30 \mathrm{~dB} / \mathrm{V}$ have been measured. A detailed study of the nonlinearity and the spurious-free dynamic range (SFDR) is presented. By optimizing the bias voltage and the input optical power, the SFDR can be improved by 10-30 dB. After minimizing the third-order distortion, an SFDR as high as $128 \mathrm{~dB}-\mathrm{Hz}^{4 / 5}$ is achieved at 10 GHz. A simple link measurement was made using this EAM and an erbium-doped fiber amplifier and a $50-\Omega$ terminated photodetector. At $10 \mathrm{GHz}$, a link gain of $1 \mathrm{~dB}$ is achieved at a detected photocurrent of $7.6 \mathrm{~mA}$ with higher gains at lower frequencies. The dependence of link gains on bias voltage, input optical, and radio frequency powers are investigated in detail.
\end{abstract}

Index Terms-Analog optical link, electroabsorption modulator (EAM), link gain, multiple quantum wells (MQWs), spurious-free dynamic range (SFDR), traveling wave (TW).

\section{INTRODUCTION}

$\mathbf{E}$ XTERNAL modulators have attracted increasing attention for both digital and analog fiber-optic links, since they can provide high speed, high efficiency, low chirp, and other features desirable for these applications. Two types of external modulators have been extensively studied: Mach-Zehnder modulators (MZMs) [1] and electroabsorption modulators (EAMs) [2]. MZMs are based on electrooptic (EO) effect. By modulating the electric field applied to one or both arms of an MZM, an index change is induced that causes the phase modulation. $\mathrm{LiNbO}_{3}$ MZMs are the most widely commercialized modulators. The electroabsorption modulator is a reverse-biased $\mathrm{p}-\mathrm{i}-\mathrm{n}$ diode with bulk active region [2] or multiple quantum wells (MQWs) [3] as the absorption layer. Electrical fields applied perpendicular to the active layer shift the absorption edge to lower photon energies. Compared with bulk absorption layers, the absorption edge

Manuscript received April 30, 2003; revised August 25, 2003.

B. Liu, A. Keating, J. Piprek, and J. E. Bowers are with the Department of Electrical and Computer Engineering, University of California, Santa Barbara CA 93106 USA (e-mail: liu@ece.ucsb.edu).

J. Shim is with the Department of Electrical and Computer Engineering, Hanyang University, 425-791 Kyungki-do, Korea, and also with the Department of Electrical and Computer Engineering, University of California, Santa Barbara, CA 93106 USA.

Y.-J. Chiu was with the Department of Electrical and Computer Engineering, University of California, Santa Barbara, CA 93106 USA. He is now with the Institute of Electro-Optical Engineering, National Sun Yat-Sen University, 804 Taiwan, R.O.C.

Digital Object Identifier 10.1109/JLT.2003.819799 in MQWs is much sharper and moves faster with the reverse-biased electric field. An MQW EAM has the advantages of low driving voltage, broad bandwidth, compact size, and monolithic integration capability with other devices. It has great potential as a low-power-consumption and low-cost external modulator for both digital and analog communications.

For digital applications, low driving voltage, large bandwidth, high extinction ratio, and low optical insertion loss are among the critical parameters to characterize a modulator [4], [5]. Compared with MZMs, MQW EAMs have the lowest driving voltage, the highest extinction ratio, and similar bandwidth. The advantages of EAMs come from two factors: high-quality strain-compensated MQWs and traveling-wave (TW) electrode. Initially, EAMs were based on bulk absorption material and lumped electrode structure. By using MQWs as the absorption layer, the quantum-confined Stark effect (QCSE) enhances the absorption edge shift with the applied electrical field and favors low drive voltage and high extinction ratio. Adopting a TW electrode not only achieves large bandwidth by removing the parasitic limit in a lumped EAM but also reduces the driving voltage and increases the extinction ratio by extending the absorption length.

For analog applications, the link gain and the dynamic range are two additional critical parameters for a modulator. Link gain depends on the optical power and the slope efficiency of the modulators. In EO-effect-based MZMs, link gain can be obtained by simply launching as high an optical input power as possible without the use of electrical or optical amplifiers. MZMs are capable of handling several hundreds of milliwatts of continuous-wave (CW) optical power with only negligible degradation. However, the optical power launched into an EAM is limited to tens of milliwatts due to the heating related to the photogenerated current and other factors. In addition, EAMs have another inherent issue that is a relative high optical insertion loss. Even with negligible coupling loss, the material absorption loss at zero bias is not negligible, since the QCSE is effective only near the exciton resonance where a substantial residual absorption always exists. To achieve a link gain, the use of an EAM with an optical amplifier is necessary; even EAMs have a high slope efficiency.

The nonlinearity of a modulator causes distortions and reduces the dynamic range of a radio frequency (RF) link. In an EO MZM, the transfer curve is well known as a sinusoidal function and is relatively independent of the input optical and RF signal powers. Therefore, the nonlinear transfer curves can be characterized accurately, and the dynamic range can be improved by linearization techniques. On the other hand, an EAM 


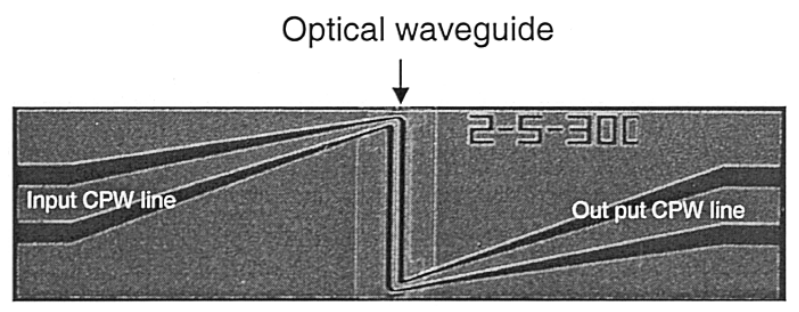

(a)

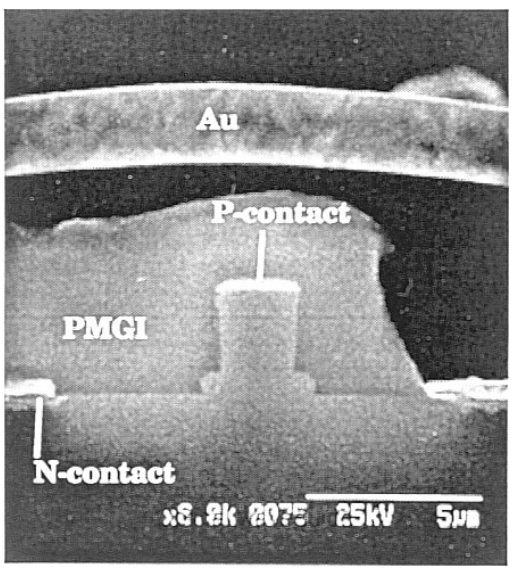

(b)

Fig. 1. (a) Top view of the TW-EAM. (b) Cross-sectional view of the EAM.

has a nonlinear transfer function that has a strong dependence on input optical wavelength and power, which could limit the dynamic range and the link gain of an RF link and makes linearization difficult. Thus, the analysis of the nonlinearity of EAMs is essential in order to enhance the dynamic range and to achieve high-quality RF links [5].

In this paper, the analog performance of InP-based TW MQW EAMs (TW-EAMs) with very low drive voltage $(<1 \mathrm{~V})$ and high slope efficiency $(>4 / \mathrm{V})$ is characterized. A detailed study of slope efficiency, spurious-free dynamic range (SFDR), and link gain with and without an erbium-doped fiber amplifier (EDFA) of a TW-EAM is conducted. This paper is organized as follows. In the next section, the structure and the fabrication of TW-EAMs will be described. In Section III, the characteristics including transfer curves under different optical powers, optical wavelengths, and EO response will be given. In Section IV, a detailed investigation of suboctave SFDR will be conducted. The link gain, the dependence of link gain on bias, input optical, and RF powers, and the SFDR with an EDFA will be investigated in Section V. Finally, discussions and conclusions will be drawn in Sections VI and VII.

\section{DEVICE STRUCTURE AND FABRICATION}

A scanning electron microscope picture of the top view of a TW EAM is shown in Fig. 1(a). Fig. 1(b) shows the detailed structure of a cross-sectional view of a TW-EAM. The epistructure is an InGaAsP-based material grown by metal organic chemical vapor deposition (MOCVD) on a semi-insulating InP substrate. It consists of a $0.5-\mu \mathrm{m} n+$ InP layer for bottom n-contact, an etch stop layer (20-nm-thick, 1.1- $\mu \mathrm{m} \mathrm{n}+\mathrm{InGaAsP}$ ), a 0.3- $\mu \mathrm{m} \mathrm{n}$ InP cladding layer, an MQW active region, a $1.5-\mu \mathrm{m} \mathrm{p}$ InP cladding layer, and a $0.1-\mu \mathrm{m} \mathrm{p}+$
InGaAs layer for p-contact. The active region consists of ten tensile-strained InGaAsP wells (12 nm, 0.35\% strain, bandgap $0.78 \mathrm{eV}$ ) and $11 \mathrm{InGaAsP}$ compressively strained barrier ( $7 \mathrm{~nm}, 0.57 \%$ strain, bandgap $1.042 \mathrm{eV}$ ). The total thickness of intrinsic absorption layer is about $300 \mathrm{~nm}$. The photoluminence (PL) peak is around $1510 \mathrm{~nm}$.

To improve the slope efficiency and optical saturation, several design changes are made compared with previous EAMs [6]. The most significant change is the shift of PL peak wavelength from 1490 to $1510 \mathrm{~nm}$, and this low wavelength detuning will enhance the extinction ratio [7]. The quatum-well (QW) thickness is increased from 10 to $12 \mathrm{~nm}$, which will enhance the QCSE effect. At the same time, the barrier heights are lowered compared with our previous design. The electron barrier is reduced from 0.13 to $0.115 \mathrm{eV}$, the heavy hole barrier is reduced from 0.163 to $0.147 \mathrm{eV}$, and the light hole barrier is reduced from 0.231 to $0.212 \mathrm{eV}$. Because of the shift of the absorption peak wavelength closer to the operation wavelength, the thick QW, and the low barriers, the required internal field needed to achieve a large absorption change is reduced. Thus, we expect this design will give low operating voltage, high slope efficiency, and high saturation power. However, the insertion loss will be increased with the small wavelength tuning.

The device fabrication [6] starts from depositing p-type ohmic contact ( $\mathrm{Ti}-\mathrm{Pt}-\mathrm{Au}$ ) with a width of $2 \mu \mathrm{m}$. The contact is then used as a mask to etch the optical ridge waveguide by $\mathrm{CH}_{4}-\mathrm{H}_{2}-\mathrm{Ar}$-based reactive ion etch (RIE). A laser interferometry method is used to accurately control the etching depth to stop right before the n-type contact layer. Wet chemical etch $\left(\mathrm{H}_{3} \mathrm{P} \mathrm{O}_{4}: \mathrm{HCl}\right)$ is followed to smooth the side wall, remove the residual n-type InP, and reduce the RIE damage. Then, the n-type contact ( $\mathrm{Ni}-\mathrm{AuGe}-\mathrm{Ni}-\mathrm{Au})$ is deposited. Following it, wet etching is performed to remove all residual conductive layers and etch down to the SI-InP substrate to lay down the microwave feedlines later. Polymethylglutarimide (PMGI) is then used to passivate the etched sidewall and to reduce the capacitance from the p-electrode. PMGI bridges are formed on both ends of the optical waveguide to connect grounds from two sides of the optical waveguide. Finally, a thick layer of $\mathrm{Au}$ $(>2 \mu \mathrm{m})$ is deposited for better microwave conductivity.

\section{MODUlator CHARACTERISTICS}

The typical normalized fiber-to-fiber dc transmission curve of our MQW TW-EAMs with a length of $300 \mu \mathrm{m}$ operated at a wavelength of $1555 \mathrm{~nm}$ is shown in Fig. 2(a). Fig. 2(b) is the transmission curve in log scale normalized to the input power. The input optical power is $2 \mathrm{dBm}$. The modulation efficiency is higher than $30 \mathrm{~dB}$ from 0 to $1 \mathrm{~V}$. This is the best reported modulation efficiency for a TW-EAM, which is mainly attributed to the improved strain-compensated QW design. For analog links, the slope efficiency is critical for RF gain. Fig. 2(a) also plots the dc slope efficiency of the normalized transfer curve. The EAM has a very high slope of $>4 / \mathrm{V}$ around zero bias. For the purposes of comparison with MZMs, an equivalent voltage $\mathrm{V}_{\pi \text {,eq }}$, defined by $\mathrm{V}_{\pi, \text { eq }}=(\pi / 2)\left|(\partial T) /\left(\partial \mathrm{V}_{\mathrm{RF}}\right)\right|^{-1}$ (where $T$ is the normalized transmission, $\mathrm{V}_{\mathrm{RF}}$ is the RF voltage), can be used to express the slope efficiency of EAMs [5]. Therefore, our EAM 


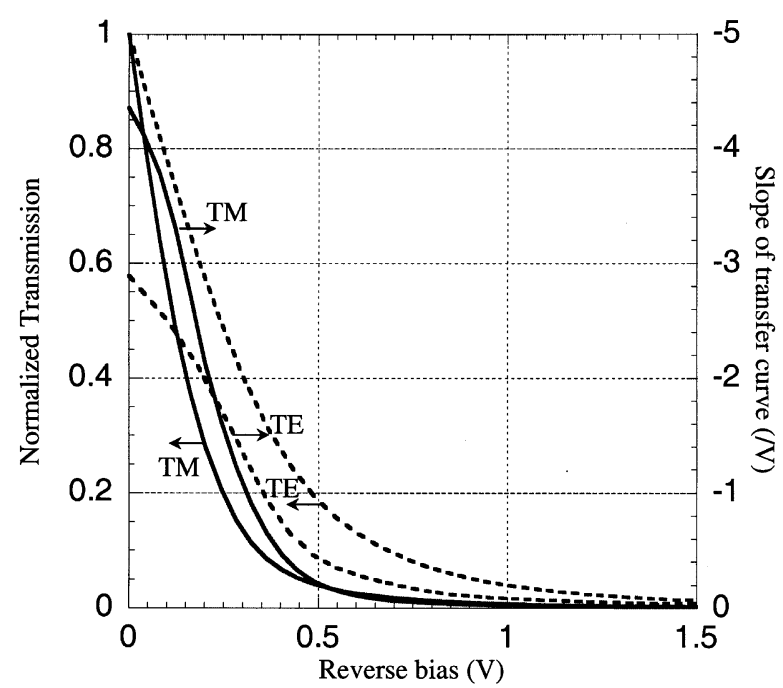

(a)

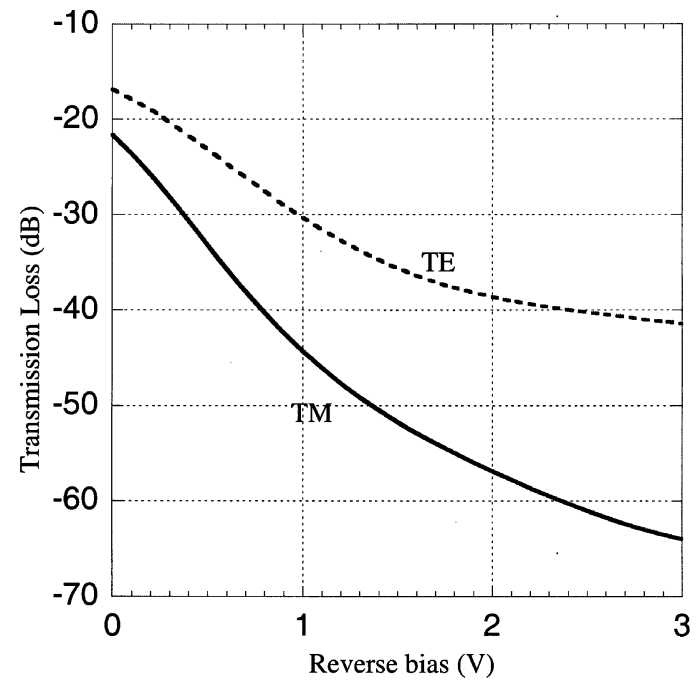

(b)

Fig. 2. (a) Normalized transfer curve and the slope for a TW-EAM. (b) Measured transfer curves in log scale.

has a $\mathrm{V}_{\pi}$ as low as $0.37 \mathrm{~V}$, which is the lowest $\mathrm{V}_{\pi \text {,eq }}$ ever reported.

Compared with EO MZMs, the performance of EAMs is much more sensitive to optical wavelength and the input optical power, especially for modulators with a QW absorption region. Fig. 3(a) shows the normalized transfer curves at different wavelengths with an input power of $2 \mathrm{~mW}$. As we expected, MQW EAMs have different slope efficiencies and extinction ratios at different wavelengths. The dependence of slope efficiency on wavelength is plotted in Fig. 3(b). The slope increases when the wavelength moves to long wavelength and then decreases when the wavelength moves away from the band edge. The peak slope efficiency is about $4 / \mathrm{V}$, which happens around $1553 \mathrm{~nm}$. Because of this strong wavelength dependence, the linearity of EAMs can be improved by tuning the input light wavelength, and the dynamic range can be enhanced [8].

The input optical power also affects the performance of EAMs due to carrier pileup and band-filling effects. Fig. 4 plots the transfer curves under different optical input power

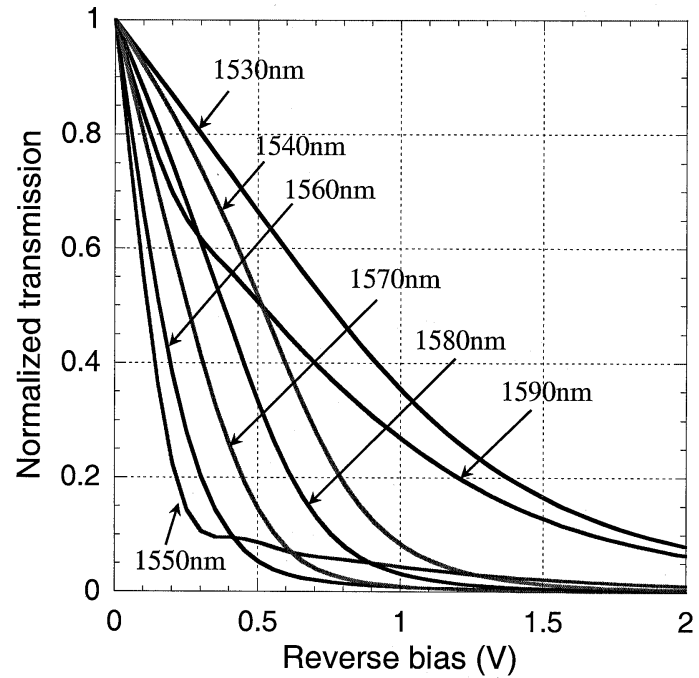

(a)

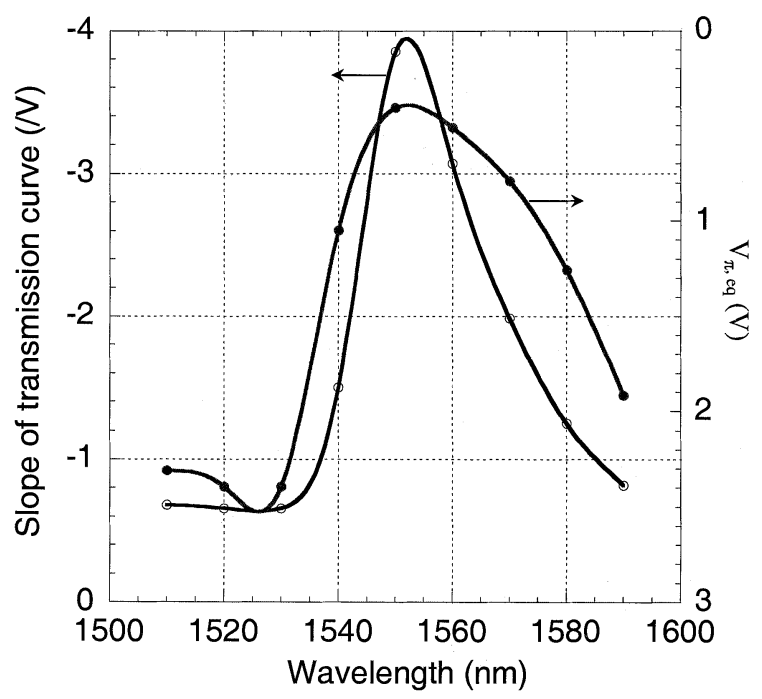

(b)

Fig. 3. (a) Normalized transfer curve at different optical wavelengths. (b) Slope efficiency and the equivalent $\mathrm{V}_{\pi}$ at zero bias.

with the wavelength of $1553 \mathrm{~nm}$. Both the slope efficiency and the linearity of the transfer curves change with the input power. At low input powers, MQW EAMs have the maximum slope efficiency. With the increase of the input power, the slope decreases. This is a substantial difference from MZMs, which are almost independent of optical power. Thus, the RF gain in an EAM modulated link will not be perfectly proportional to the input optical power with a slope of two. Fig. 5 shows the measured RF output power versus the input optical power at $10 \mathrm{GHz}$. The slope of the curve is smaller than two, as we expected. However, as we will show subsequently, the linearity of EAMs is improved with the increase of the input power.

\section{SFDR}

One of the important performance parameters for high-speed analog communication is the SFDR [5]. In externally modulated fiber-optic links, the dynamic range is usually dominated by the distortion of the external optical modulator. 


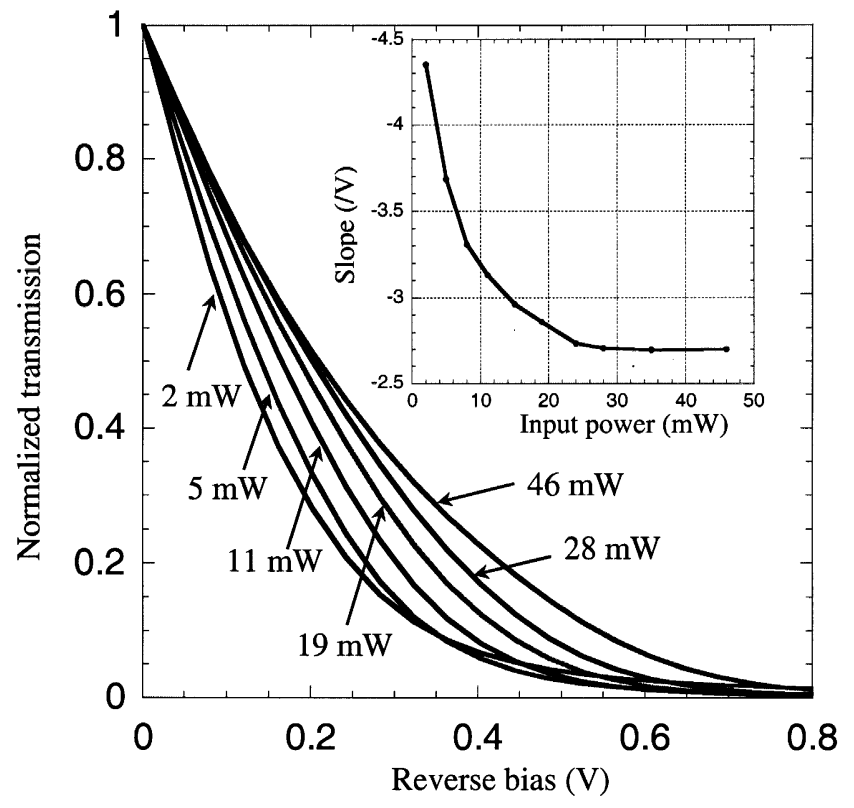

Fig. 4. Normalized transfer curve at different input optical power. The insert is the slope efficiency at zero bias.

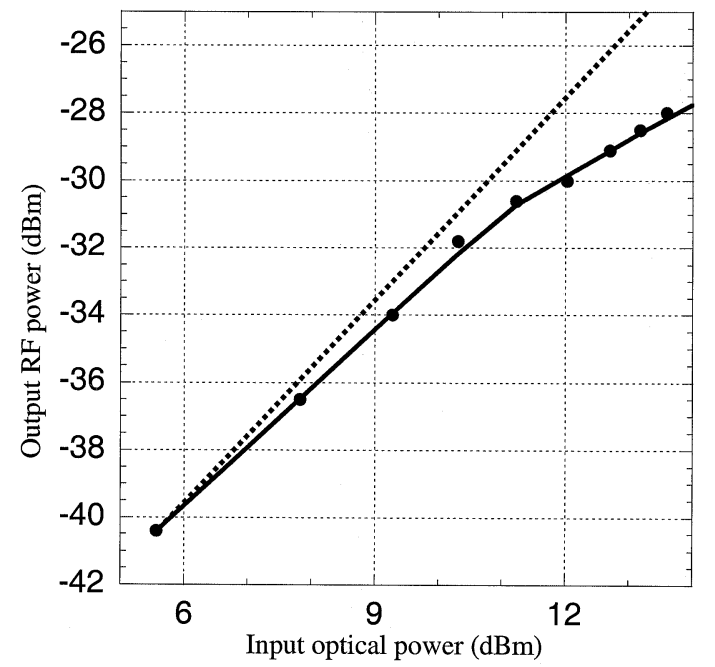

Fig. 5. Measured output RF power versus the input optical power. The dashed line has a slope of two, which is the case in MZMs.

It is well known that the third-order intermodulation product can be minimized and a high SFDR can be achieved if a modulator is biased at the null point of the third derivative of the transfer curve [9]. For EAMs, the bias dependence of the SFDR is more sensitive due to the exponential transfer function. Fig. 6 plots the third derivatives of the measured dc transfer functions of an MQW TW-EAM for the transverse magnetic (TM) mode at different input optical powers. It is clear that there is a null point in each transfer curve, and the corresponding bias depends on the input optical power. Furthermore, the third-order derivative decreases and the linearity improves with the increase of the input optical power. Thus, we expect that the third-order distortion can be minimized and a high dynamic range can be achieved by adjusting both bias voltage and the input optical power. Although tuning the input optical wavelength is also an alternative to minimize the third-order distortion [8], adjusting the input

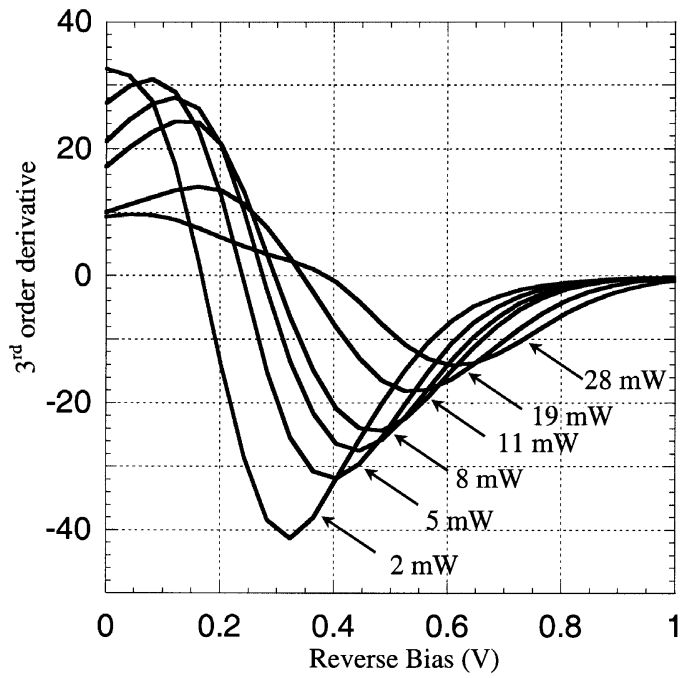

Fig. 6. Third-order derivatives of the transfer function of an EAM under different input optical power.

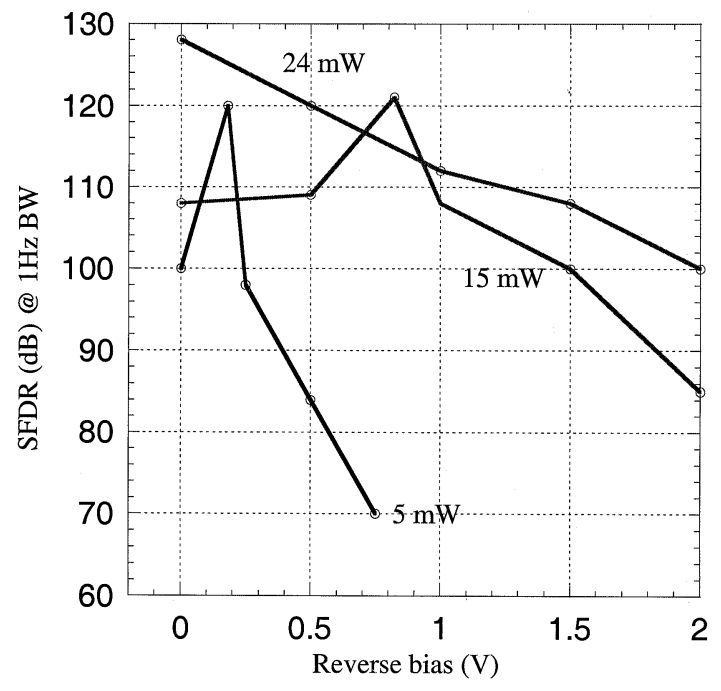

Fig. 7. Measured SFDR in a $1-\mathrm{Hz}$ bandwidth versus bias with different input optical powers.

optical power is a cost-effective and simple way tp achieve this purpose. To further improve the SFDR, the fifth-order distortion also needs to be reduced. If we check the fifth derivatives of the transfer curves, we do find that both third- and fifth-order derivatives can be simultaneously zero by carefully adjusting the input optical power and the polarization.

The two-tone method was used to investigate the suboctave SFDR of our TW-EAMs at $10 \mathrm{GHz}$. Two RF signals from synthesizers are combined and fed into a modulator. The frequencies of the two tones are set to 10 and $9.9 \mathrm{GHz}$. The third-order intermodulation products are observed at 10.1 and $9.8 \mathrm{GHz}$. A high-power distributed feedback (DFB) laser with wavelength at $1553 \mathrm{~nm}$ is used as the light source. The DFB laser has a low relative intensity noise (RIN) of $\sim 160 \mathrm{~dB} / \mathrm{Hz}$ with a high power operation at $10 \mathrm{GHz}$. A highly linear photodetector is used to receive the output signals.

The measured suboctave SFDR results at different biases and different input optical powers are summarized in Fig. 7. By adjusting the bias voltage, 10-20-dB dynamic range improvement 


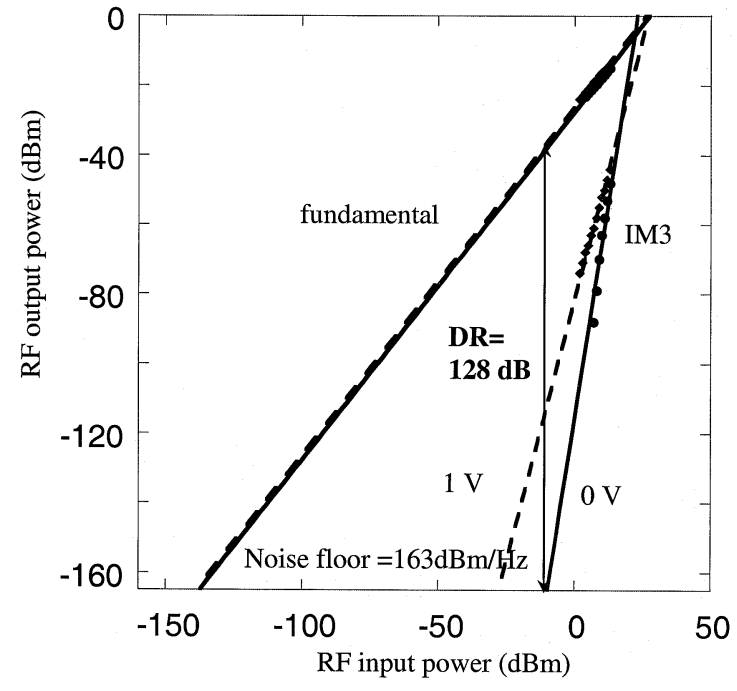

Fig. 8. Measured fundamental and third-order intermodulation signals.

can be achieved. By checking the intermodulation signals, the slope of the intermodulation product versus the input RF signal is five at the peak SFDR point, where the third-order derivative is zero. Fig. 8 shows the measured fundamental and the intermodulation RF signals at two different bias points with 24-mW transverse electric (TE) optical input power. At 0-V bias with an average photocurrent of $0.21 \mathrm{~mA}$, the slope of the intermodulation signal is $>5$ and the highest SFDR of $128 \mathrm{~dB}-\mathrm{Hz}^{4 / 5}$ is achieved when we fit the linear curve with a slope of five (the best fitted curve has a slope of 6.5, and this increases the dynamic range by $5 \mathrm{~dB}$ ). Interestingly, the intermodulation signal at this optimized point does not have a uniform slope. The slope changes from nine to five with increasing input RF power. This phenomenon has been theoretically predicted and experimentally observed in linearized EO modulators [10], [11]. However, since we could not measure the intermodulation signal down to the noise floor, a leveling off or even a rise of the intermodulation signal is not observed [11]. When the bias is increased to $1 \mathrm{~V}$, the slope of the intermodulation product is three and the corresponding SFDR is reduced to $110 \mathrm{~dB}-\mathrm{Hz}^{2 / 3}$. Thus, the third-order nonlinearity dominates the distortion. The most interesting observation here is that the dynamic range and the third-order derivative null point strongly depend on the input optical power level, as we expected from the measured dc transfer curves. When the optical power increases from 6 to $24 \mathrm{~mW}$, SFDR increases by $15-30 \mathrm{~dB}$.

For an EAM with low driving voltage and high extinction ratio, $\mathrm{RF}$ saturation phenomena are anticipated. This is due to the nonlinear characteristic of the transfer curve. Fig. 9 shows the RF output power versus the RF input power under different bias voltages. Sublinear, linear, and superlinear curves are clearly observed for large RF input power under different bias voltages, respectively. Theoretical simulations show good agreement with the experiments.

\section{LINK GAIN AND SFDR WITH AN EDFA}

For an ideal externally modulated optical link, the link gain can be simply expressed by [12] $g=s_{m}^{2} s_{d}^{2}\left(R_{\text {load }}\right) /\left(R_{m}\right)$,

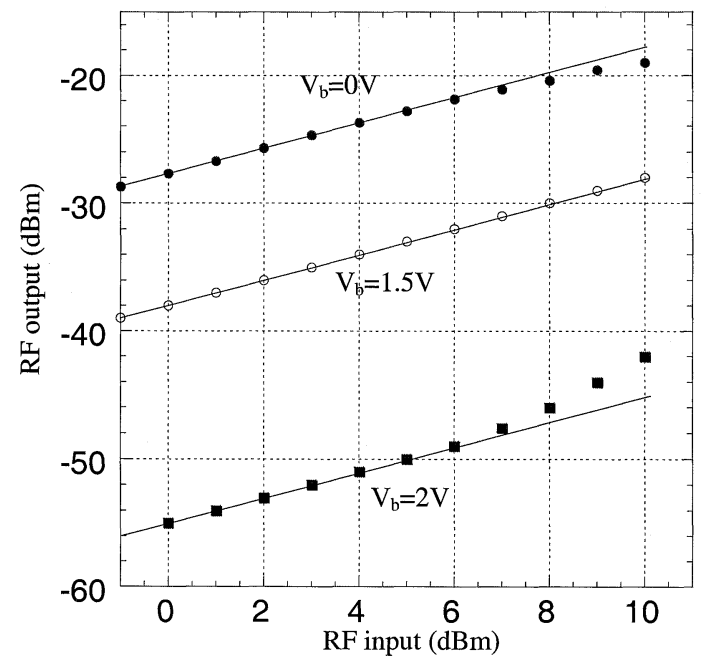

Fig. 9. RF output power versus RF input power at different biases.

where $s_{m}$ and $s_{d}$ are the quantum slope efficiencies of the modulator and the photodetector, respectively, and $R_{\text {load }}$ and $R_{m}$ are the load impedance and the impedance of the modulator. For an EAM, the slope efficiency is given by $s_{m}=\left(\pi T_{0} P_{L} R_{m}\right) /\left(2 V_{\pi, \text { eq }}\right)$, where $T_{0}$ is the modulator's input-fiber-to-output-fiber insertion loss and $P_{L}$ is the input CW optical power; $\mathrm{V}_{\pi \text {,eq }}$ has been defined previously.

Thus, it is clear that launching the input optical power as high as possible is the simplest way to improve the link gain in an externally modulated optical analog link. This method has been widely adopted for EO MZMs. However, very high optical power is unacceptable for an EAM for two reasons.

1) The slope efficiency of an EAM declines with the increase of the optical power, as we observed previously. Thus, we cannot expect the link gain to follow the increases of the optical power with a slope of two, and the link gain will saturate at high optical power.

2) Similar to other semiconductor devices, the maximum optical power that an EAM can handle is limited due to the facet damage and the heating from photocurrent. Furthermore, EAMs have very high optical insertion loss (small $T_{0}$ ) when we use a wavelength near the band edge.

Table I is a survey of the link performance based on EAMs from the publications. Clearly, there is a long way to go to reach 0-dB link gain for an EAM without amplification. To get a link gain $>0 \mathrm{~dB}$ in an EAM modulated link, amplification is unavoidable. Compared with electrical amplifiers, optical amplifiers such as EDFAs are more efficient and cost-effective for the transmission of high-frequency signals. In this section, we will demonstrate an EAM modulated optical link with an EDFA to compensate the optical loss and boost the link gain. As a result of this work, a link gain of $1 \mathrm{~dB}$ has been achieved.

In the link experiment with an EDFA, the EDFA is inserted between the EAM and detector to amplify the modulated optical signal. A tunable optical filter is placed before the detector to remove excess spontaneous emission. The link gain without an EDFA is shown in Fig. 10 under different biases and input optical power, where the input RF signal is $-10 \mathrm{dBm}$ at $10 \mathrm{GHz}$. With $10 \mathrm{dBm}$ optical input, the link gain is only about $-40 \mathrm{~dB}$. 
TABLE I

SurVey of the Performances of EAM Modulated ANALOg OPTICAL LinKS

\begin{tabular}{l|l|l|l|l}
\hline & $\begin{array}{l}\text { Link gain } \\
(\mathrm{dB})\end{array}$ & $\begin{array}{l}\text { Average } \\
\text { photocurrent }(\mathrm{mA})\end{array}$ & $\begin{array}{l}\text { Subocatve } \\
\text { SFDR } \\
\left.\mathrm{Hz}^{4 / 5}\right)\end{array}$ & $\begin{array}{l}\text { Operation } \\
\text { frequency } \\
(\mathrm{GHz})\end{array}$ \\
\hline Sun [14] & -32 & 0.25 & 123 & 0.78 \\
\hline Welstand [15] & -17.4 & 3 & 123 & 4 \\
\hline Loi [16] & -25.5 & 0.98 & 114 & 0.9 \\
\hline Kaneko [17] & $\begin{array}{l}-20.6 \\
(10.3 \text { with } \\
\text { matching circuit })\end{array}$ & 4.56 & 123 & 5.6 \\
\hline $\begin{array}{l}\text { Li [18] } \\
\text { This work w/o } \\
\text { EDFA }\end{array}$ & -35 & $\sim 1$ & & 18 \\
\hline $\begin{array}{l}\text { This work w/ } \\
\text { EDFA }\end{array}$ & -35 & 0.2 & 128 & 10 \\
\hline
\end{tabular}

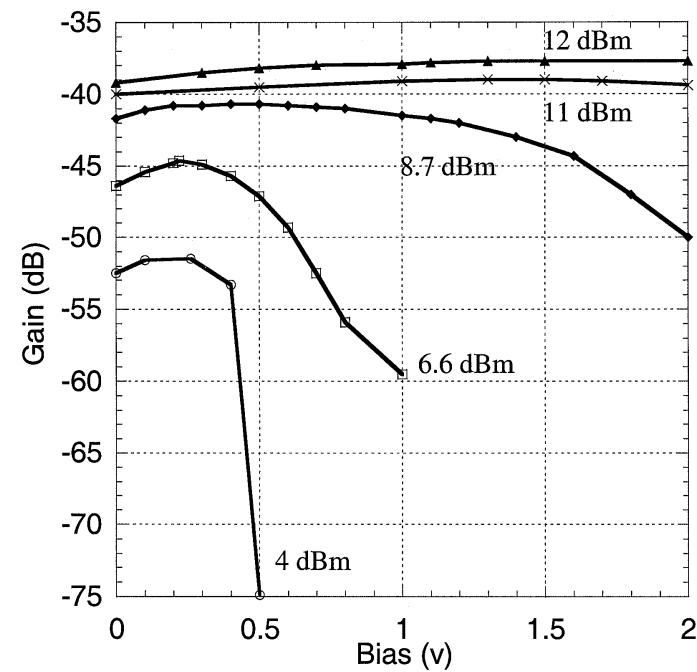

Fig. 10. Link gain versus bias at different optical input power.

The main limitation is from the high optical insertion loss, which is about $18 \mathrm{~dB}$ at $0-\mathrm{V}$ bias for this EAM used in our experiment. The best link gain without an EDFA for an EAM with 15-dB insertion loss is about $-30 \mathrm{~dB}$ at $14-\mathrm{dBm}$ optical input. From Fig. 10, we also observed that the dependence of link gain on the bias is much stronger at low input optical power than at high input optical power. This is because the linearity is improved with the increase of the input optical power. However, the slope efficiency is degraded and the link gain starts to saturate, which is expected from the de transfer curve characteristics.

Fig. 11 is the link gain with an EDFA as a function of the photocurrent of the detector. The input RF signal is $-26 \mathrm{dBm}$ at $10 \mathrm{GHz}$, the input optical power is about $5.5 \mathrm{dBm}$, and the wavelength is $1555 \mathrm{~nm}$. The bias voltage is $0.5 \mathrm{~V}$ for the TM mode and $0.95 \mathrm{~V}$ for the TE mode. At a photocurrent of $7 \mathrm{~mA}$, the link gain reaches $0 \mathrm{~dB}$ for the TM mode, which corresponds to an EDFA gain of $25 \mathrm{~dB}$. A link gain of $1 \mathrm{~dB}$ is achieved at a photocurrent of $7.6 \mathrm{~mA}$. The maximum link gain is limited by the saturation of photodetector. Compared with the TM mode, the TE mode has a 2-dB lower link gain; this is due to the smaller slope efficiency of the TE mode.

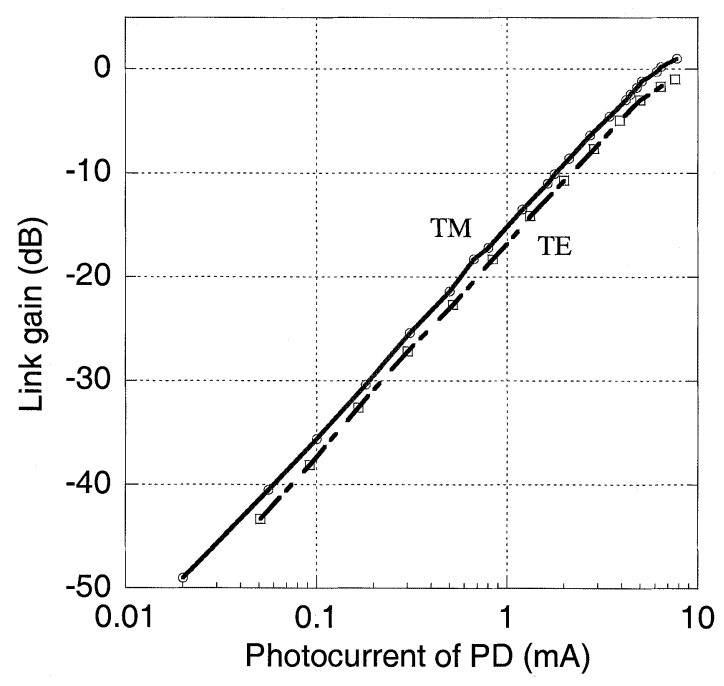

Fig. 11. Link gain with an EDFA versus the average photocurrent.

Fig. 12(a) and (b) shows the measured link gain and the noise figure as a function of the EAM bias for both (a) TM and (b) TE modes. At the highest link gain of $1 \mathrm{~dB}$, the corresponding NF is about $34 \mathrm{~dB}$. The noise sources for a link using EDFA include the thermal noises associated with the RF generator and the receiver, the RIN of the laser, the shot noise from the photodetector, and the noise associated with EDFA. In our link setup, the laser RIN at $10 \mathrm{GHz}$ is about $165 \mathrm{~dB} / \mathrm{Hz}$ and the corresponding noise is about $-160 \mathrm{dBm} / \mathrm{Hz}$. The shot noise from the photodiode is about $-159 \mathrm{dBm} / \mathrm{Hz}$. In the link without an EDFA, the $\mathrm{NF}$ is about $50 \mathrm{~dB}$, which is dominated by the link loss. Adding an EDFA causes signal-spontaneous beat noise, which dominates the noise figure [13]. However, the noise figure has been improved by $16 \mathrm{~dB}$, which is due to the link gain.

The optical and RF saturations are also investigated in our link. Fig. 13 shows the link gain versus the input optical power. The saturation is due to the slope efficiency decrease and the saturation of the EDFA and detector. Fig. 14 is the RF output power as a function of the RF input power. The RF saturation power is about $-5 \mathrm{dBm}$. 


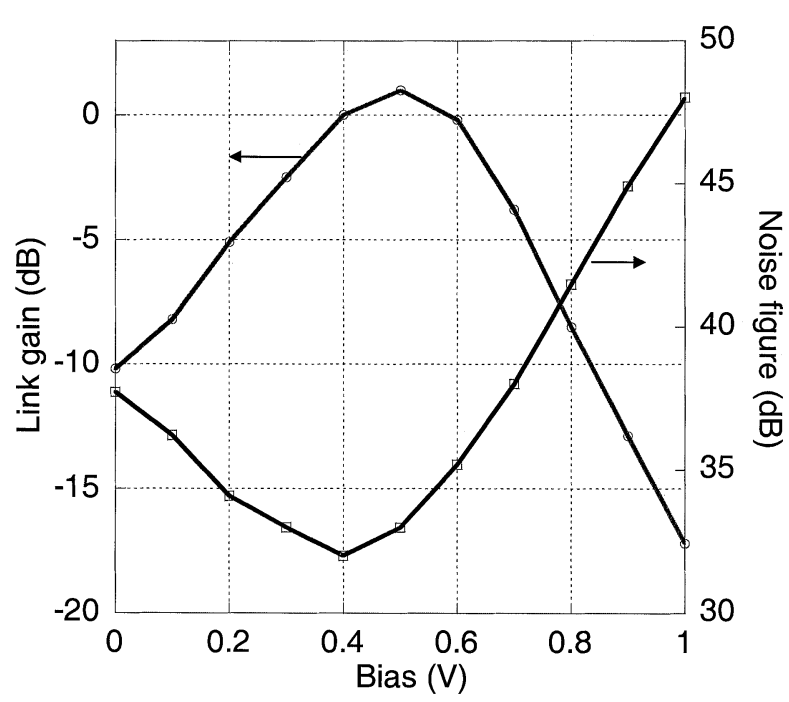

(a)

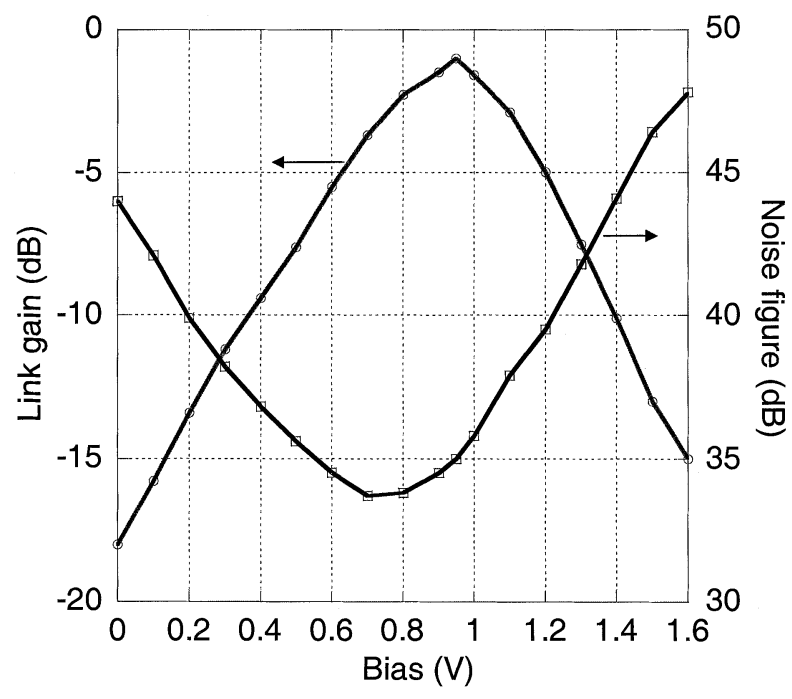

(b)

Fig. 12. Link gain and noise figure versus the bias: (a) TM mode and (b) TE mode.

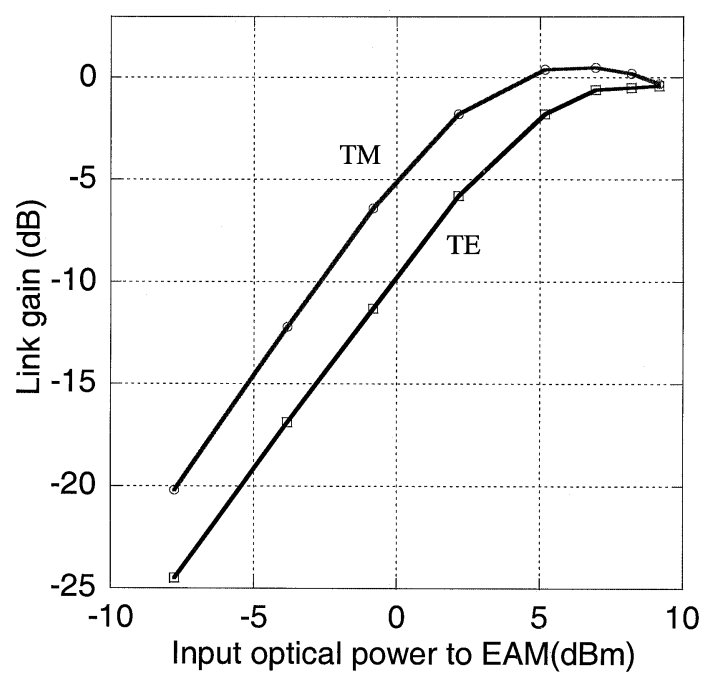

Fig. 13. Link gain versus the input optical power in a link with an EDFA.

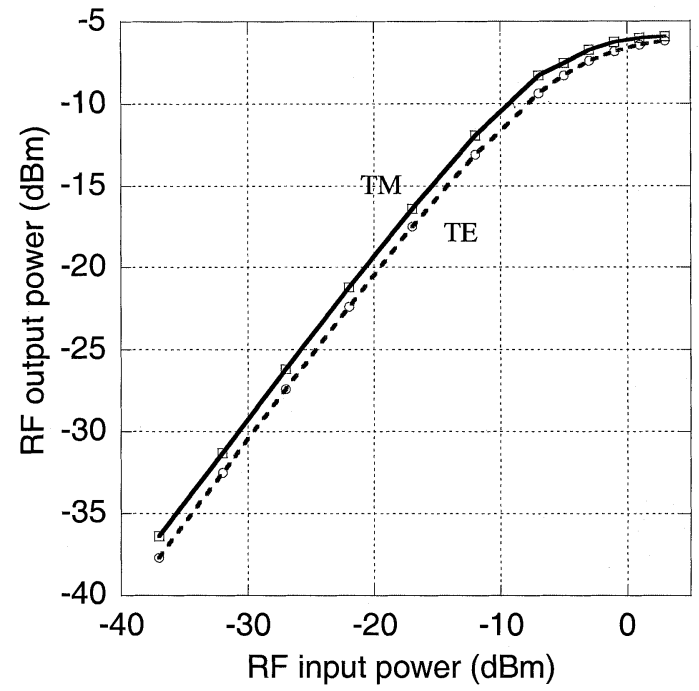

Fig. 14. Link gain and RF output power versus RF input power.

The suboctave SFDR is measured for a link using a TW-EAM and an EDFA. Fig. 15(a) and (b) shows the measured fundamental and the intermodulation RF signals at two different bias points. One corresponds to the bias to maximize the SFDR and another corresponds to the bias to maximize the link gain. At the bias of $0.52 \mathrm{~V}$, the measured SFDR is about $112 \mathrm{~dB}-\mathrm{Hz}^{4 / 5}$ at $1-\mathrm{Hz}$ bandwidth. Thus, the third-order distortion has been minimized at this bias. Compared with a link without an EDFA, the SFDR is decreased. This is mainly due to the noise floor's increasing more than $20 \mathrm{~dB}$. At the bias of $0.46 \mathrm{~V}$, the link has the maximum link gain of $1 \mathrm{~dB}$; however, the SFDR is decreased to $102 \mathrm{~dB}-\mathrm{Hz}^{2 / 3}$. The third-order distortion dominates the dynamic range here. Note that the link gain at maximum SFDR bias is only about $1 \mathrm{~dB}$ lower than the maximum gain, but the dynamic range improvement is $10 \mathrm{~dB}$.

\section{Discussions}

Although InP-based TW-EAMs have demonstrated many superior characteristics over EO MZMs, especially low drive voltage, high modulation efficiency, wide bandwidth, compact size, and low cost, there are still several issues that need to be solved for high-performance analog optic links. The first is the optical loss. Using EDFA is a cost-effective way to compensate the loss, but the noise figure will be degraded due to the spontaneous noise. To improve both link gain and NF, reducing the insertion loss is necessary. The goal is to achieve the optical insertion loss close to the value of $\mathrm{LiNbO}_{3} \mathrm{MZMs}$, which is about $4-6 \mathrm{~dB}$. The second is to further improve SFDR and achieve high SFDR over a wide operation range. We have measured SFDR as high as $128 \mathrm{~dB}-\mathrm{Hz}^{4 / 5}$ without an EDFA and $112 \mathrm{~dB}-\mathrm{Hz}^{4 / 5}$ with an EDFA. However, it is achieved under a specific bias. The optimized bias point depends on the input optical power, wavelength, and RF power and frequency, which complicate the system implementation. Thus, improving SFDR over a broadened operation range is necessary.

To reduce the insertion loss, reducing the residual optical absorption as low as possible and improving the waveguide fabrication to lower the scattering loss are important. Integrating 


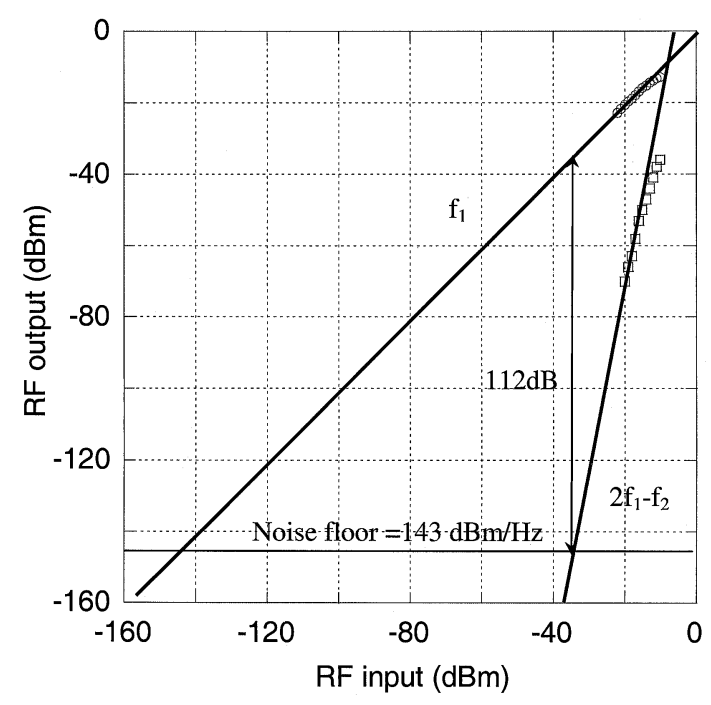

(a)

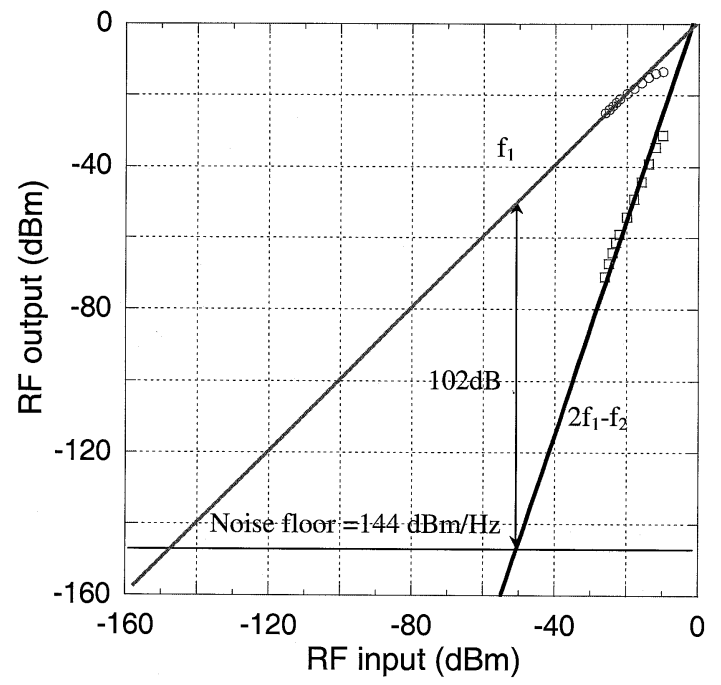

(b)

Fig. 15. Measured fundamental and third-order intermodulation signals versus RF input power in a link with an EDFA: (a) $0.52-\mathrm{V}$ and (b) $0.46-\mathrm{V}$ bias voltage.

mode converters with EAMs is an efficient way to improve the coupling efficiency. The current coupling loss for both facets is about $7.5 \mathrm{~dB}$. With two mode converters, the coupling loss can be reduced to $3 \mathrm{~dB}$.

To achieve a high SFDR, linearization techniques are needed. Although the ideas used for linearizing EO modulators can be borrowed directly, such as the dual-parallel approach, electronic predistortion, and feed-forward, the more promising way is the all-optical linearization technique, since it has the potential of low cost and avoids expensive electrical circuits-for example, dual-wavelength modulation if we can integrate a tunable laser source with EAM for linearity enhancement.

\section{CONCLUSION}

In conclusion, we have successfully fabricated high-performance TW MQW EAMs for analog applications. A high slope efficiency of $>4 / \mathrm{V}$ at $1.55 \mu \mathrm{m}$ for a $300-\mu \mathrm{m}$-long EAM is measured, which is equivalent to an MZM with a $\mathrm{V}_{\pi}$ of $0.37 \mathrm{~V}$. By adjusting the bias voltage as well as the input optical power, the increase of the SFDR can be as much as $30 \mathrm{~dB}$. An SFDR as high as $128 \mathrm{~dB}-\mathrm{Hz}^{4 / 5}$ at $10 \mathrm{GHz}$ has been achieved. With the help of EDFA, a link gain of $>0 \mathrm{~dB}$ at $10 \mathrm{GHz}$ has been successfully demonstrated. The dependence of link gain on the bias, the input RF, and optical power have been investigated in detail. Compared with a link without EDFA, the link gain is improved by $50 \mathrm{~dB}$, and the noise figure is improved by $16 \mathrm{~dB}$ with an EDFA. By further reducing the optical insertion loss and improving the linearity, MQW TW-EAMs will be cost-effective external intensity modulators for high-speed analog communications.

\section{REFERENCES}

[1] R. C. Alferness, "Waveguide electrooptic modulators," IEEE Trans. Microwave Theory Tech., vol. MTT-30, pp. 1121-1137, Aug. 1982.

[2] C. Rolland, K. L. Prosyk, C. M. Maritan, and N. Puertz, "High speed and low loss, bulk electroabsorption waveguide modulators at $1.3 \mathrm{~m}$," IEEE Photon. Technol. Lett., vol. 3, pp. 894-896, Oct. 1991.

[3] D. A. B. Miller, T. C. Damen, A. C. Gossard, W. Wiegmann, T. H. Wood, and C. A. Burus, "Band-edge electroabsorption in quantum well structures: the quantum-confined Stark effect," Phys. Rev. Lett., vol. 53, pp. 2173-2175, 1984.

[4] R. G. Walker, "High-speed III-V semiconductor intensity modulators," IEEE. J. Quantum Electron., vol. 27, pp. 654-667, Mar. 1991.

[5] W. S. C. Chang, "Multiple quantum well electroabsorption modulators for RF photonic links," in RF Photonic Technology in Optical Fiber Links, W. S. C. Chang, Ed. Cambridge, U.K.: Cambridge Univ. Press, 2002, pp. 133-162.

[6] S. Z. Zhang, P. Abraham, and J. E. Bowers, "25 GHz polarization-insensitive electroabsorption modulators with traveling-wave electrodes," IEEE Photon. Technol. Lett., vol. 11, pp. 191-193, Feb. 1999.

[7] K. Yamada, Y. Matsui, T. Kunii, and Y. Ogawa, "Negative-chirp electroabsorption modulator using low-wavelength tuning," IEEE Photon. Technol. Lett., vol. 7, pp. 1157-1158, Oct. 1995.

[8] K. K. Loi, X. B. Mei, C. W. Tu, and W. S. C. Chang, "Linearization of 1.3- $\mu \mathrm{m}$ MQW electroabsorption modulators using an all-optical frequency-insensitive technique," IEEE Photon. Technol. Lett., vol. 10, pp. 964-966, July 1998.

[9] R. B. Welstand, S. A. Pappert, Y. Z. Liu, J. M. Chen, J. T. Zhu, A. L. Kellner, and P. K. L. Yu, "Enhanced linear dynamic range property of Franz-Keldysh effect waveguide modulator," IEEE Photon. Technol. Lett., vol. 7, pp. 751-753, July 1995.

[10] W. B. Bridges and J. H. Schaffner, "Distortion in linearized electrooptic modulators," IEEE Trans. Microwave Theory Tech., vol. 43, pp. 2184-2197, Sept. 1995.

[11] G. E. Betts and F. J. O'Donnell, "Microwave analog optical links using suboctave linearized modulators," IEEE Photon. Technol. Lett., vol. 8, pp. 1273-1275, Sept. 1996.

[12] E. I. Ackerman and C. H. Cox, "RF fiber-optic link performance," IEEE Microwave Mag., pp. 50-58, Dec. 2001.

[13] G. E. Betts, "LiNbO3 external modulators and their use in high performance analog link," in RF Photonic Technology in Optical Fiber Links, W. S. C. Chang, Ed. Cambridge, U.K.: Cambridge Univ. Press, 2002, pp. 81-129.

[14] C. K. Sun, R. B. Welstand, J. T. Zhu, P. K. L. Yu, Y. Z. Liu, and J. M. Chen, "High spurious free dynamic range fiber link using a semiconductor electroabsorption modulator," Electron. Lett., vol. 31, pp. 902-903, 1995.

[15] R. B. Welstand, C. K. Sun, J. T. Zhu, Y. Z. Liu, and P. K. L. Yu, "Dualfunction electroabsorption waveguide modulator/detector for optoelectronic transceiver applications," IEEE Photon. Technol. Lett., vol. 8, pp. 1540-1542, Nov. 1996.

[16] K. K. Loi, X. B. Mei, C. W. Tu, W. S. C. Chang, D. T. Nichols, L. J. Lembo, and J. C. Brock, "Low-loss 1.3- $\mu \mathrm{m}$ MQW electroabsorption modulators for high-linearity analog optical links," IEEE Photon. Technol. Lett., vol. 10, pp. 1572-1574, Nov. 1998.

[17] S. Kaneko, Y. Miyazaki, H. Watanabe, K. Kasahara, and T. Tajime, "An electroabsorption modulator module for digital and analog applications," J. Lightwave Technol., vol. 17, pp. 669-676, Apr. 1999. 
[18] G. L. Li, P. Mages, C. K. Sun, W. S. C. Chang, and P. K. L. Yu, "High-saturation high-speed traveling-wave InGaAsP-InP electroabsorption modulator," IEEE Photon. Technol. Lett., vol. 13, pp. 1076-1078, Oct. 2001.
Yi-Jen Chiu, photograph and biography not available at the time of publication.

Adrian Keating (S'90-M'90), photograph and biography not available at the time of publication.

Joachim Piprek (M'94-SM'98), photograph and biography not available at the time of publication.

John E. Bowers, photograph and biography not available at the time of publication. 